\title{
Transmission and reflection features of all-dielectrics metasurfaces with electric and magnetic resonances
}

Terekhov, Pavel, Babicheva, Viktoriia, Baryshnikova, Kseniia, Shalin, Alexander, Karabchevsky, Alina, et al.

Pavel D. Terekhov, Viktoriia E. Babicheva, Kseniia V. Baryshnikova, Alexander S. Shalin, Alina Karabchevsky, Andrey B. Evlyukhin, "Transmission and reflection features of all-dielectrics metasurfaces with electric and magnetic resonances," Proc. SPIE 10927, Photonic and Phononic Properties of Engineered Nanostructures IX, 109270I (4 March 2019); doi: 10.1117/12.2506973 


\title{
Transmission and reflection features of all-dielectrics metasurfaces with electric and magnetic resonances
}

\author{
Pavel D. Terekhove,b,c,d, Viktoriia E. Babicheva ${ }^{\mathrm{e}}$, Kseniia V. Baryshnikova ${ }^{\mathrm{b}}$, Alexander S. \\ Shalin $^{\mathrm{b}}$, Alina Karabchevskya,c,d, and Andrey B. Evlyukhin ${ }^{\mathrm{b}, \mathrm{f}, \mathrm{g}}$ \\ ${ }^{a}$ Electrooptics and Photonics Engineering Department, Ben-Gurion University, Beer-Sheva \\ 8410501, Israel \\ ${ }^{b}$ ITMO University, 49 Kronversky Ave., 197101, St. Petersburg, Russia \\ ${ }^{\mathrm{c}}$ Ilse Katz Institute for Nanoscale Science \& Technology, Ben-Gurion University, Beer-Sheva \\ 8410501, Israel \\ ${ }^{\mathrm{d}}$ Center for Quantum Information Science and Technology, Ben-Gurion University, Beer-Sheva \\ 8410501, Israel \\ eUniversity of Arizona, Tucson, Arizona 85721, USA \\ ${ }^{\mathrm{f}}$ Moscow Institute of Physics and Technology, 9 Institutsky Lane, Dolgoprudny 141700, Russia \\ ${ }^{\mathrm{g}}$ Institute of Quantum Optics, Leibniz Universität Hannover, 30167 Hannover, Germany
}

\begin{abstract}
The effective multipole decomposition approach is applied to study the optical features of the silicon metasurface in the near-infrared. The spectral regions of perfect transmission and reflection have been analyzed using the Cartesian multipole decomposition. It is shown that transmission peaks appear due to the mutual interaction of multipole moments up to the third order, while reflection peaks are due to the dominant contribution of one of the multipole moments. The results of this work can be broadly applied to design novel metasurfaces, sensors, and optical filters.
\end{abstract}

Keywords: Metasurface, multipole, silicon, dielectric, infrared

\section{INTRODUCTION}

Dielectric nanophotonics is the intensively studied area of modern science with high potential for device applications. Optical properties of dielectric nanostructures attract significant attention of scientific groups over the world. ${ }^{1-4}$ Dielectric materials provide the opportunity to manipulate both electric and magnetic components of light using the simple particle geometries. In other words, such sub-wavelength particles support the excitation of strong electric and magnetic multipole resonances. These resonances can be used to control light scattering by changing material, shape, and aspect ratio of nanoparticles. ${ }^{5,6}$ The mutual interaction between multipole moments can be used in a wide range of applications such as nanoantennas, ${ }^{7,8}$ sensing, ${ }^{9-12}$ cloaking, ${ }^{13,14}$ solar cell technology ${ }^{15}$ and functional metasurfaces. ${ }^{16,17}$ Moreover, the multipole decomposition approach has recently been applied to analyze dielectric structures in the terahertz frequency range ${ }^{18}$ and even macroscopic objects like the Great Pyramid. ${ }^{19}$

In this work, we study the periodic array of silicon nanocubes embedded in air. We study the optical properties of the structure using a multipole decomposition approach and analyze the features of transmission and reflection spectra. Obtained results can be used to design novel optical sensors and filters and multi-functional metasurfaces for the near-infrared.

Further author information:

P.D.T.: E-mail: terekhovpd@gmail.com

Photonic and Phononic Properties of Engineered Nanostructures IX, edited by Ali Adibi,

Shawn-Yu Lin, Axel Scherer, Proc. of SPIE Vol. 10927, 1092701 · C 2019 SPIE

CCC code: $0277-786 X / 19 / \$ 18 \cdot$ doi: $10.1117 / 12.2506973$

Proc. of SPIE Vol. 10927 109270I-1 


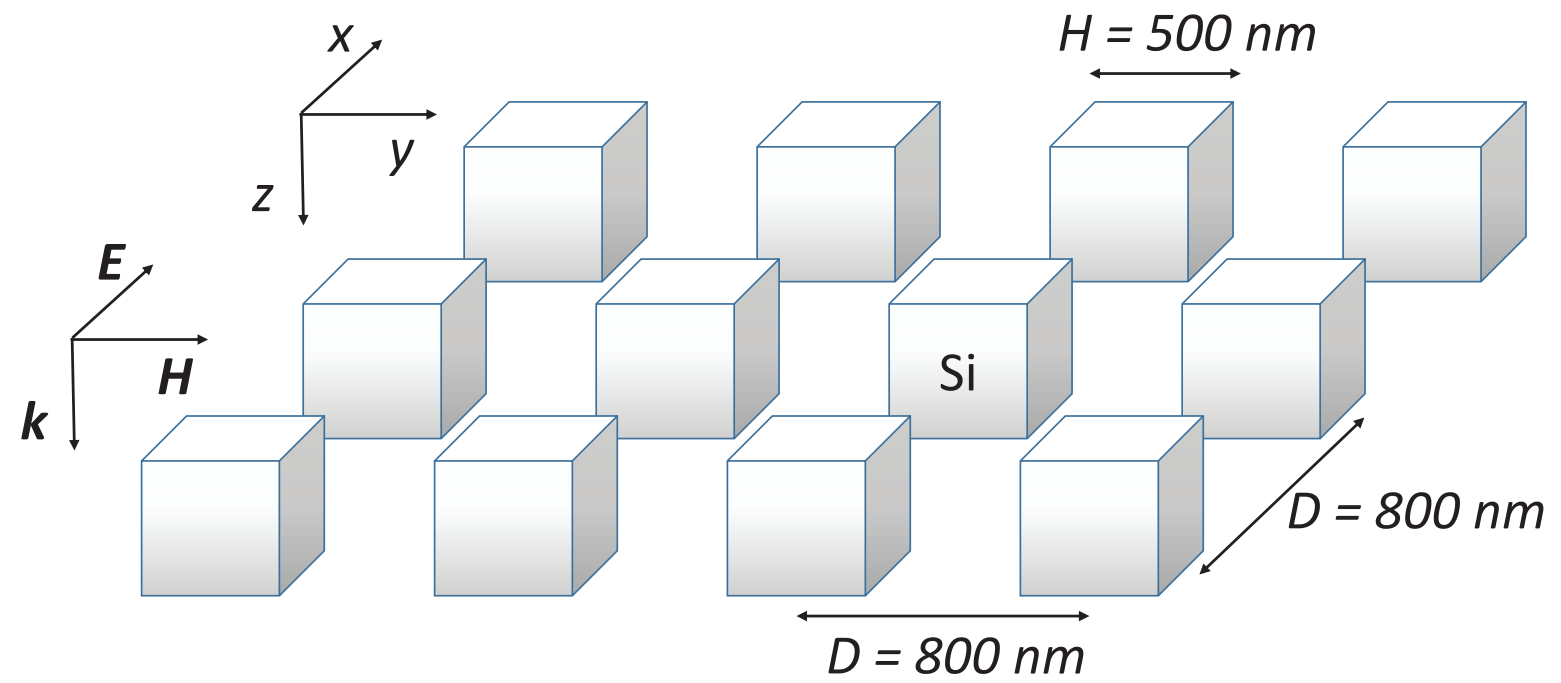

Figure 1. The artistic representation of the studied system.

\section{THEORETICAL BACKGROUND}

Here we use the multipole decomposition approach reported in Ref. ${ }^{20}$ to study the cubic silicon metasurface in near-infrared. The infinite two-dimensional (2D) array of nanocubes is placed in (xy) plane and illuminated with a linearly polarized plane wave with $x$-polarization.

In this case, the electric field reflection $r$ and transmission $t$ coefficients of the array can be written as ${ }^{20}$

$$
\begin{gathered}
r=\frac{i k_{d}}{E_{0} 2 S_{L} \varepsilon_{0} \varepsilon_{d}}\left(p_{x}-\frac{1}{v_{d}} m_{y}+\frac{i k_{d}}{6} Q_{x z}-\frac{i k_{d}}{2 v_{d}} M_{y z}-\frac{k_{d}^{2}}{6} O_{x z z}\right), \\
t=1+\frac{i k_{d}}{E_{0} 2 S_{L} \varepsilon_{0} \varepsilon_{d}}\left(p_{x}+\frac{1}{v_{d}} m_{y}-\frac{i k_{d}}{6} Q_{x z} \frac{i k_{d}}{2 v_{d}} M_{y z}-\frac{k_{d}^{2}}{6} O_{x z z}\right),
\end{gathered}
$$

where $k_{d}=k_{0} \sqrt{\varepsilon_{d}}$ is the wavenumber in a surrounding medium ( $k_{0}$ is the wavenumber in vacuum), $S_{L}$ is the area of a lattice unit cell $\left(S_{L}=D^{2}\right.$ for the square lattice, where $D$ is the lattice constant), $\varepsilon_{0}$ is the vacuum permittivity, $\varepsilon_{d}$ is the permittivity of a surrounding medium, $v_{d}=1 / \sqrt{\mu_{0} \varepsilon_{0} \varepsilon_{d}}$ is the speed of light in the surrounding nonmagnetic medium, $p_{x}$ and $m_{y}$ are the $x$ and $y$ components of total electric (TED) and magnetic dipole (MD) moments, respectively, $Q_{x z}, M_{y z}$ and $O_{x z z}$ are the corresponding components symmetrized and traceless tensors of electric quadrupole (EQ), magnetic quadrupole (MQ), and electric octupole (EOC) moments, respectively. ${ }^{21}$

The reflection and transmission coefficients are

$$
R=|r|^{2}, \quad T=|t|^{2} .
$$

Then the absorption coefficient $A$ could be derived from the following expression $A=1-R-T$.

In next section, we numerically prove that the multipole expressions for $T$ and $R$ obtained above are in an excellent agreement with the direct numerical calculations of the transmission and reflection for periodic dielectric metasurfaces. 


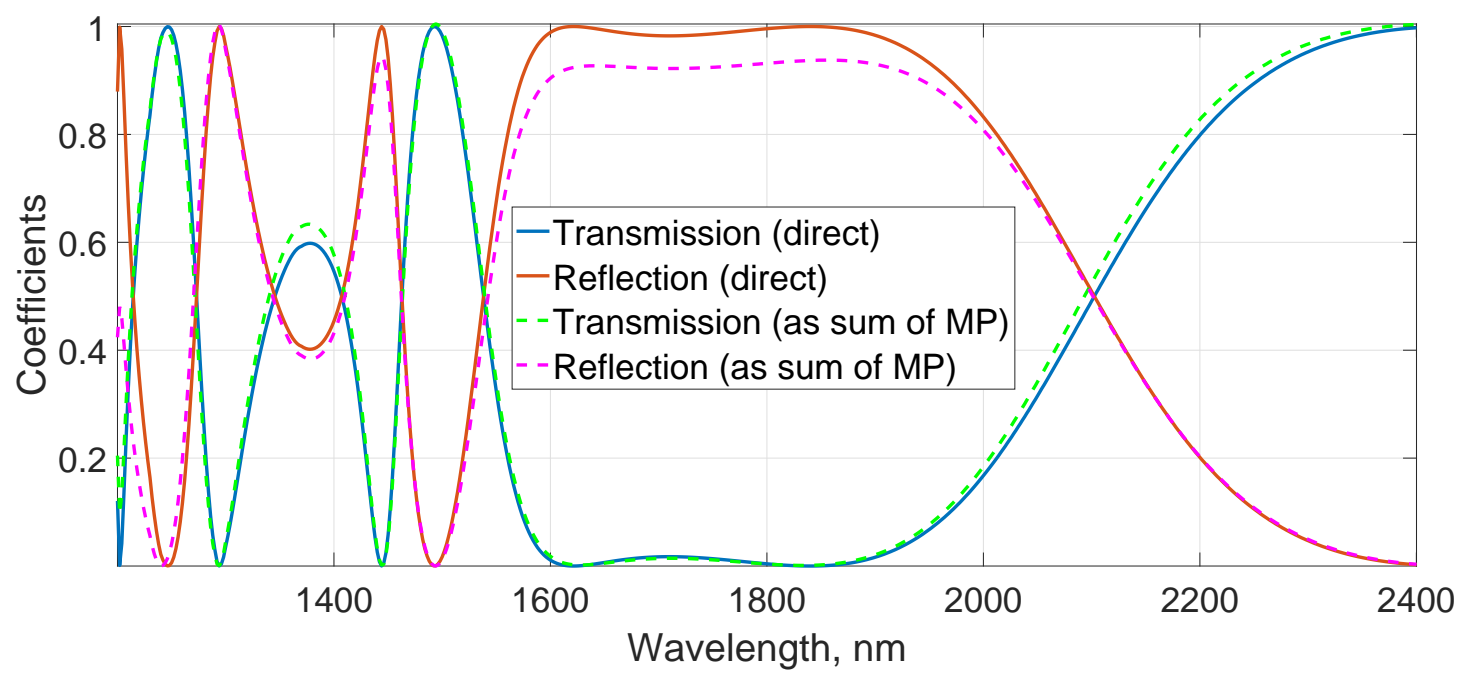

Figure 2. Spectra of the transmission $T$, reflection $R$, and absorption $A$ coefficients of the metasurfaces composed of silicon nanocubes of height $H=500 \mathrm{~nm}$ with period $D=800 \mathrm{~nm}$

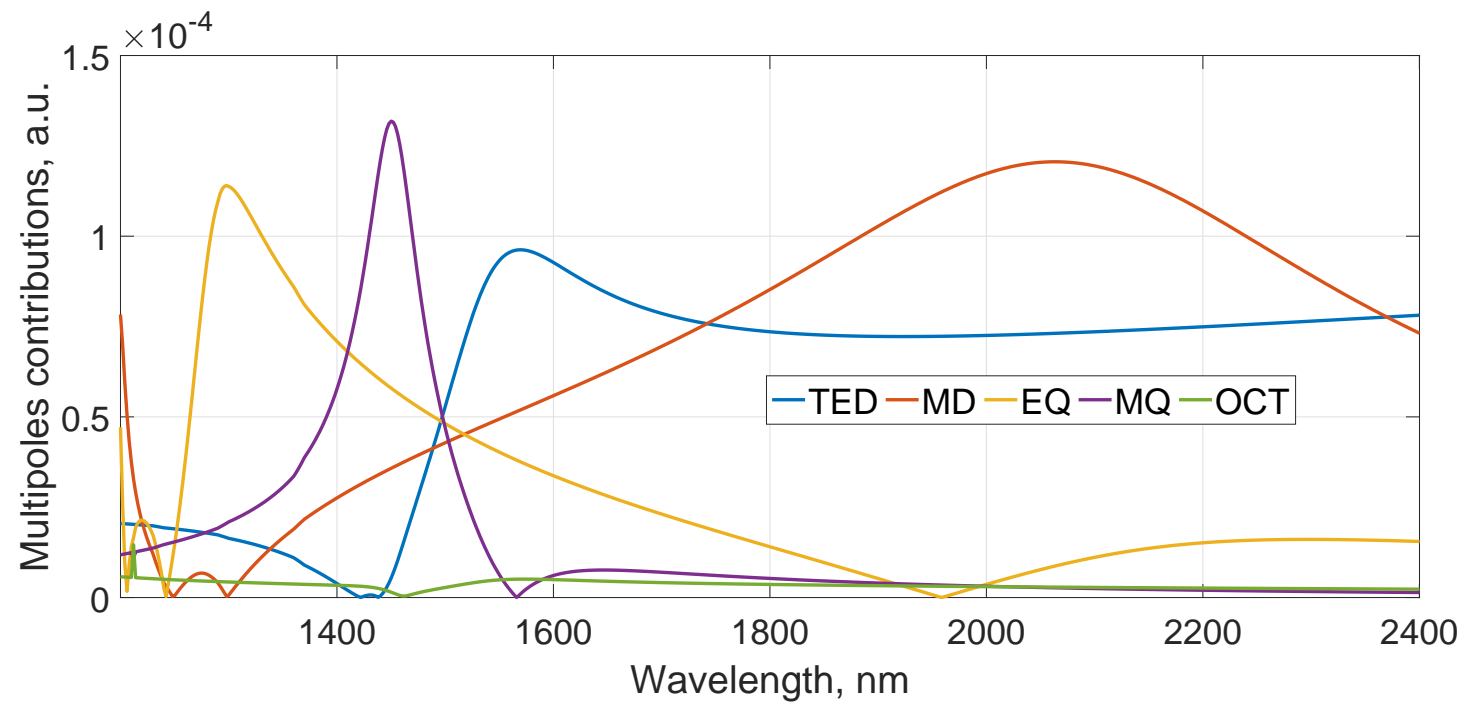

Figure 3. Absolute values of the multipole contributions in the electric field reflection and transmission coefficients $r$ and $t$ of the metasurfaces composed of silicon nanocubes of height $H=500 \mathrm{~nm}$ with period $D=800 \mathrm{~nm}$

\section{RESULTS AND DISCUSSION}

Here we apply the multipole decomposition approach to study the optical properties of the metasurface in Fig. 1. The base edge of each nanocube is equal to $H=500 \mathrm{~nm}$ and the lattice constant is $D=800 \mathrm{~nm}$.

Fig. 2 presents the transmission and reflection spectra of the metasurface in near-infrared. Note that absorption can be neglected due to transparency of silicon in the considered range. The direct calculations of transmission and reflection parameters are in the excellent agreement with the results obtained with multipole decomposition approach (Eq. 3). We note three wavelengths of the perfect transmission through the metasurface $(\lambda \approx 1246 \mathrm{~nm}, \lambda \approx 1494 \mathrm{~nm}$ and $\lambda \approx 2400 \mathrm{~nm})$ and four wavelengths of the perfect reflection $\operatorname{appears}(\lambda \approx 1294 \mathrm{~nm}, \lambda \approx 1444 \mathrm{~nm}, \lambda \approx 1622 \mathrm{~nm}$ and $\lambda \approx 1844 \mathrm{~nm})$. Fig. 3 presents the absolute values of 


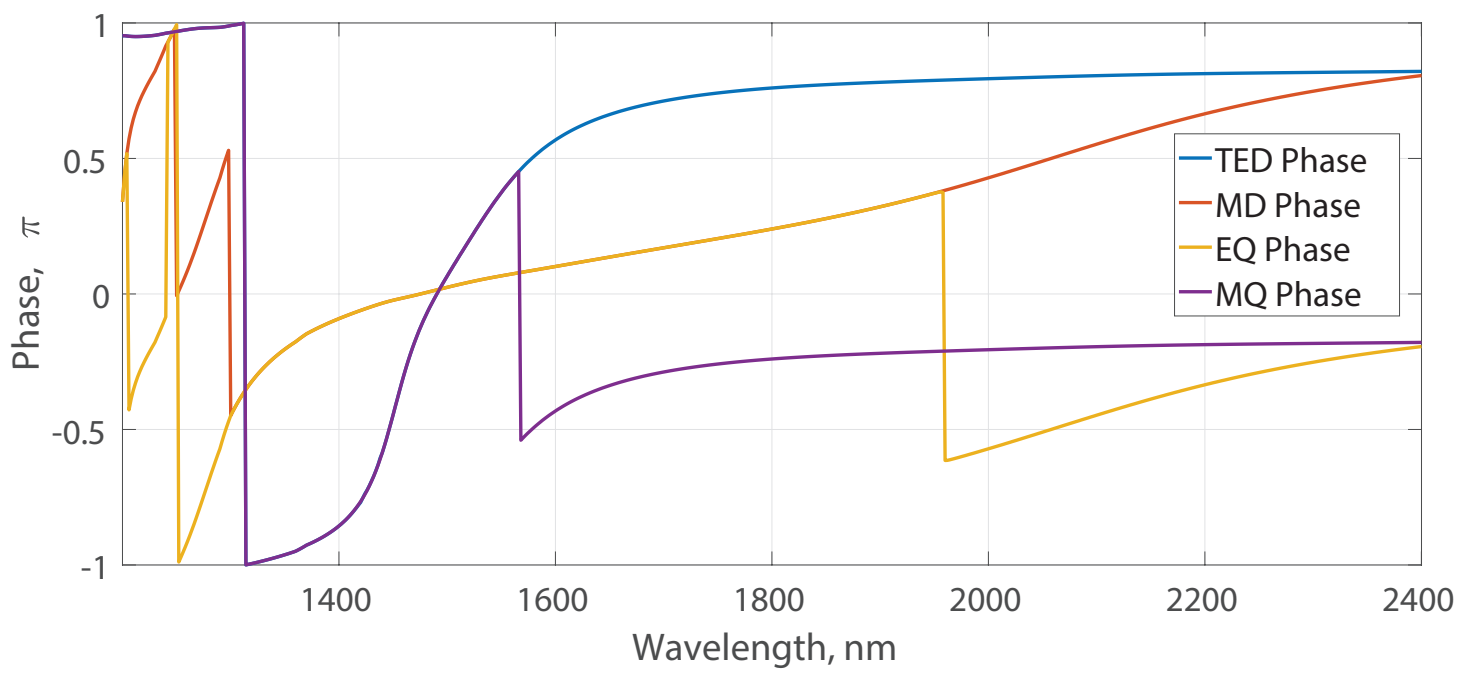

Figure 4. Phases of the multipole terms in the amplitude reflection and transmission coefficients of the metasurface composed of nanocubes of height $H=500 \mathrm{~nm}$ with period $D=800 \mathrm{~nm}$

the multipole moment contributions to the electric field reflection and transmission coefficients $r$ and $t$ for the considered metasurface. We use this multipole analysis together with the phases of multipole contributions (Fig. 4) to study the origins of optical properties of the metasurface.

The interesting case of perfect transmission can be seen at $\lambda \approx 1494 \mathrm{~nm}$. The contributions of TED, MD, EQ, and MQ moments in the $r$ and $t$ coefficients are approximately equal at this wavelength (Fig. 3). Moreover, the phases of these contributions are also equal to each other (Fig. 4). Similar conditions provide homogeneous side scattering for the single particle ${ }^{22,23}$ and so-called lattice invisibility effect for the infinite array. ${ }^{20}$ In turn, at $\lambda \approx 1294 \mathrm{~nm}$ multipole contributions are not equal to each other; TED and MQ provide dominant contribution at this wavelength. The phases of multipole contributions are equal as well as for $\lambda \approx 1494 \mathrm{~nm}$ (Fig. 4). At $\lambda \approx 2400 \mathrm{~nm}$ usual Kerker conditions ${ }^{24,25}$ fulfillment leads to the perfect transmission through the metasurface.

As for the perfect reflection features of the studied metasurface, there are two sharp peaks at $\lambda \approx 1294 \mathrm{~nm}$ and $\lambda \approx 1444 \mathrm{~nm}$ and the region of high reflection with two perfect reflection spectral points at $\lambda \approx 1622 \mathrm{~nm}$ and $\lambda \approx 1844 \mathrm{~nm}$ (Fig. 3). Every peak is provided mainly by one multipole moment contribution: EQ for $\lambda \approx 1622 \mathrm{~nm}$, MQ for $\lambda \approx 1444 \mathrm{~nm}$, TED for $\lambda \approx 1622 \mathrm{~nm}$ and MD for $\lambda \approx 1844 \mathrm{~nm}$. It is worth noting that for $\lambda \approx 1622 \mathrm{~nm}$ and $\lambda \approx 1844 \mathrm{~nm}$ phase difference between TED and MD moments is equal to $\pi / 2$ (Fig. 4, and it corresponds to the forward scattering suppression according to Kerker conditions.

\section{CONCLUSIONS}

In this work, we utilized the multipole decomposition approach to analyze the cubic silicon metasurface in the near-infrared. We studied transmission and reflection features to explain their origins with multipole theory. It has been shown that transmission peaks mainly provided due to the mutual interaction of different multipole moments. In contradiction, reflection peaks appear due to the dominant contribution of TED, MD, EQ or MQ moment.

Multipole decomposition of transmission and reflection spectra provides the useful tool for semi-analytical analysis of arrays of non-spherical nanoparticles in near-infrared spectral range. Our results can be used to design novel photonic devices, sensors, and optical applications. 


\section{ACKNOWLEDGMENTS}

This work has been supported by the Israeli Ministry of Trade and Labor-Kamin Program, Grant. No. 62045 . A.S.S acknowledges the support of the Russian Fund for Basic Research within the projects 18-02-00414, 1852-00005 and the support of the Ministry of Education and Science of the Russian Federation (GOSZADANIE Grant No. 3.4982.2017/6.7). A.B.E. acknowledges the support of Ministry of Education and Science of the Russian Federation (16.7162.2017/8.9). The development of analytical approach and the calculations of multipole moments have been supported by the Russian Science Foundation Grant No. 16-12-10287. Support has been provided by the Government of the Russian Federation (Grant No. 074-U01). The research described was performed as a part of the joint Ph.D. program between the BGU and ITMO. V.E.B. work is supported by the Air Force Office of Scientific Research under Grant No. FA9550-19-1-0032.

\section{REFERENCES}

[1] Evlyukhin, A. B., Reinhardt, C., Seidel, A., Lukyanchuk, B. S., and Chichkov, B. N., "Optical response features of si-nanoparticle arrays," Physical Review B 82(4), 045404 (2010).

[2] Kuznetsov, A. I., Miroshnichenko, A. E., Brongersma, M. L., Kivshar, Y. S., and Lukyanchuk, B., "Optically resonant dielectric nanostructures," Science 354(6314), aag2472 (2016).

[3] Jahani, S. and Jacob, Z., "All-dielectric metamaterials," Nature Nanotechnology 11(1), 23-36 (2016).

[4] Staude, I. and Schilling, J., "Metamaterial-inspired silicon nanophotonics," Nature Photonics 11(5), 274 (2017).

[5] Evlyukhin, A. B., Reinhardt, C., and Chichkov, B. N., "Multipole light scattering by nonspherical nanoparticles in the discrete dipole approximation," Physical Review B 84(23), 235429 (2011).

[6] Terekhov, P. D., Baryshnikova, K. V., Shalin, A. S., Karabchevsky, A., and Evlyukhin, A. B., "Resonant forward scattering of light by high-refractive-index dielectric nanoparticles with toroidal dipole contribution," Optics Letters 42(4), 835-838 (2017).

[7] Krasnok, A. E., Miroshnichenko, A. E., Belov, P. A., and Kivshar, Y. S., "All-dielectric optical nanoantennas," Optics Express 20(18), 20599-20604 (2012).

[8] Baryshnikova, K. V., Novitsky, A., Evlyukhin, A. B., and Shalin, A. S., "Magnetic field concentration with coaxial silicon nanocylinders in the optical spectral range," JOSA B 34(7), D36-D41 (2017).

[9] Katiyi, A. and Karabchevsky, A., "Figure of merit of all-dielectric waveguide structures for absorption overtone spectroscopy," Journal of Lightwave Technology 35(14), 2902-2908 (2017).

[10] Bontempi, N., Chong, K. E., Orton, H. W., Staude, I., Choi, D.-Y., Alessandri, I., Kivshar, Y. S., and Neshev, D. N., "Highly sensitive biosensors based on all-dielectric nanoresonators," Nanoscale 9(15), 4972 4980 (2017).

[11] Katiyi, A. and Karabchevsky, A., "Si nanostrip optical waveguide for on-chip broadband molecular overtone spectroscopy in near-infrared," ACS sensors 3(3), 618-623 (2018).

[12] Yang, C.-Y., Yang, J.-H., Yang, Z.-Y., Zhou, Z.-X., Sun, M.-G., Babicheva, V. E., and Chen, K.-P., "Nonradiating silicon nanoantenna metasurfaces as narrowband absorbers," ACS Photonics (2018).

[13] Ospanova, A. K., Labate, G., Matekovits, L., and Basharin, A. A., "Multipolar passive cloaking by nonradiating anapole excitation," Scientific reports 8(1), 12514 (2018).

[14] Galutin, Y., Falek, E., and Karabchevsky, A., "Invisibility cloaking scheme by evanescent fields distortion on composite plasmonic waveguides with si nano-spacer," Scientific Reports 7(1), 12076 (2017).

[15] Spinelli, P., Verschuuren, M., and Polman, A., "Broadband omnidirectional antireflection coating based on subwavelength surface mie resonators," Nature Communications 3, 692 (2012).

[16] Babicheva, V. E. and Evlyukhin, A. B., "Resonant lattice kerker effect in metasurfaces with electric and magnetic optical responses," Laser \& Photonics Reviews 11(6) (2017).

[17] Babicheva, V. E. and Evlyukhin, A. B., "Metasurfaces with electric quadrupole and magnetic dipole resonant coupling," ACS Photonics 5(5), 2022-2033 (2018).

[18] Terekhov, P., Baryshnikova, K., Evlyukhin, A., and Shalin, A., "Destructive interference between electric and toroidal dipole moments in tio2 cylinders and frustums with coaxial voids," in [Journal of Physics: Conference Series], 929(1), 012065, IOP Publishing (2017). 
[19] Balezin, M., Baryshnikova, K. V., Kapitanova, P., and Evlyukhin, A. B., "Electromagnetic properties of the great pyramid: First multipole resonances and energy concentration," Journal of Applied Physics 124(3), 034903 (2018).

[20] Terekhov, P. D., Babicheva, V. E., Baryshnikova, K. V., Shalin, A. S., Karabchevsky, A., and Evlyukhin, A. B., "Multipole analysis of dielectric metasurfaces composed of non-spherical nanoparticles and lattice invisibility effect," Physical Review B 99, 045424 (2019).

[21] Alaee, R., Rockstuhl, C., and Fernandez-Corbaton, I., "An electromagnetic multipole expansion beyond the long-wavelength approximation," Optics Communications 407, 17-21 (2018).

[22] Terekhov, P. D., Baryshnikova, K. V., Artemyev, Y. A., Karabchevsky, A., Shalin, A. S., and Evlyukhin, A. B., "Multipolar response of nonspherical silicon nanoparticles in the visible and near-infrared spectral ranges," Physical Review B 96(3), 035443 (2017).

[23] Lee, J. Y., Miroshnichenko, A. E., and Lee, R.-K., "Simultaneously nearly zero forward and nearly zero backward scattering objects," Optics express 26(23), 30393-30399 (2018).

[24] Kerker, M., Wang, D.-S., and Giles, C., "Electromagnetic scattering by magnetic spheres," JOSA 73(6), 765-767 (1983).

[25] Decker, M., Staude, I., Falkner, M., Dominguez, J., Neshev, D. N., Brener, I., Pertsch, T., and Kivshar, Y. S., "High-efficiency dielectric huygens' surfaces," Advanced Optical Materials 3(6), 813-820 (2015). 